\title{
Hadronic Vacuum Polarization Contribution to the Anomalous Magnetic Moments of Leptons from First Principles
}

\author{
Sz. Borsanyi, ${ }^{1}$ Z. Fodor, ${ }^{1,2,3}$ C. Hoelbling, ${ }^{1}$ T. Kawanai, ${ }^{3}$ S. Krieg, ${ }^{1,3}$ L. Lellouch,, \\ R. Malak, ${ }^{4,5}$ K. Miura, ${ }^{4,6}$ K. K. Szabo, ${ }^{1,3}$ C. Torrero, ${ }^{4}$ and B. C. Toth ${ }^{1}$ \\ (Budapest-Marseille-Wuppertal Collaboration) \\ ${ }^{1}$ Department of Physics, Bergische Universität Wuppertal, Gaussstrasse 20, D-42119 Wuppertal, Germany \\ ${ }^{2}$ Institute for Theoretical Physics, Eötvös University, Pázmány Péter sétány 1/A, H-1117 Budapest, Hungary \\ ${ }^{3}$ Jülich Supercomputing Centre, Forschungszentrum Jülich, D-52425 Jülich, Germany \\ ${ }^{4}$ CNRS, Aix-Marseille Université, Université de Toulon, CPT, UMR 7332, F-13288 Marseille, France \\ ${ }^{5}$ CNRS, CEA, Maison de la Simulation, USR 3441, F-91191 Gif-sur-Yvette Cedex, France \\ ${ }^{6}$ Kobayashi-Maskawa Institute for the Origin of Particles and the Universe, Nagoya University, Nagoya 464-8602, Japan
}

(Received 30 December 2017; published 12 July 2018)

\begin{abstract}
We compute the leading, strong-interaction contribution to the anomalous magnetic moment of the electron, muon, and tau using lattice quantum chromodynamics (QCD) simulations. Calculations include the effects of $u, d, s$, and $c$ quarks and are performed directly at the physical values of the quark masses and in volumes of linear extent larger than $6 \mathrm{fm}$. All connected and disconnected Wick contractions are calculated. Continuum limits are carried out using six lattice spacings. We obtain $a_{e}^{\mathrm{LO}-\mathrm{HVP}}=189.3(2.6)(5.6) \times 10^{-14}$, $a_{\mu}^{\mathrm{LO}-\mathrm{HVP}}=711.1(7.5)(17.4) \times 10^{-10}$ and $a_{\tau}^{\mathrm{LO}-\mathrm{HVP}}=341.0(0.8)(3.2) \times 10^{-8}$, where the first error is statistical and the second is systematic.
\end{abstract}

DOI: 10.1103/PhysRevLett.121.022002

Introduction.-Ever since the discovery of the electron's spin $[1,2]$, the magnetic moments of leptons have accompanied the development of quantum mechanics and quantum field theory. This is particularly true of the small, "anomalous," quantum corrections to these moments, $a_{\ell}$, where $\ell$ denotes either the electron $(e)$, the muon $(\mu)$ or the tau $(\tau)$ (see, e.g., Ref. [3] for an introduction). Today, $a_{e}$ is one of the most precisely measured [4] and computed [5,6] quantities in nature, with a total uncertainty below $1 \mathrm{ppb}$. Theory and experiment agree and the measurement can be used to make the most precise determination of the fine-structure constant $\alpha$ [6].

In the case of the muon, the precision of the measurement [7] and of the standard model (SM) prediction (e.g., Ref. [8]) are roughly matched at around $0.5 \mathrm{ppm}$. However, theory and experiment disagree by more than 3 standard deviations. This is particularly enticing, because it could be a sign of new, fundamental physics. The anomalous magnetic moment of the muon is generically much more sensitive to new, massive degrees of freedom than that of the electron. This is because,

Published by the American Physical Society under the terms of the Creative Commons Attribution 4.0 International license. Further distribution of this work must maintain attribution to the author(s) and the published article's title, journal citation, and DOI. Funded by SCOAP ${ }^{3}$. in many extensions of the SM, the contributions of new particles are proportional to the lepton mass squared, which is roughly $4 \times 10^{4}$ times larger for the muon. Moreover, a new experiment is beginning to take data at Fermilab [9], with the goal of reducing errors by a factor of four, and another one is planned at J-PARC, with similar objectives [10].

The same argument should make $a_{\tau}$ even more interesting for new physics searches: the $\tau$ mass is close to 17 times that of the muon. However, its very short lifetime, of order $10^{-13} s$, has meant that no direct measurement of $a_{\tau}$ has yet been made, though a concrete proposal for doing so [11] is being implemented [12].

Theoretically, the leading source of uncertainty in the SM prediction of $a_{\mu}$ is the leading order (LO) hadronic vacuum polarization (HVP) contribution, $a_{\mu}^{\mathrm{LO}-\mathrm{HVP}}$, which is responsible for over $79 \%$ of the total error [8]. This contribution also dominates the uncertainty in $a_{\tau}$ [13] and for $a_{e}$, has a total size of around 6.5 times the experimental error [14]. Today this contribution is determined most precisely using dispersion relations and the cross section of $e^{+} e^{-}$to hadrons and/or the rate of hadronic $\tau$ decays $[3,8,15-20]$. However, since the pioneering work of Ref. [21], lattice QCD calculations of $a_{\mu}^{\mathrm{LO}-\mathrm{HVP}}$ [22-39] have made significant progress and provide a completely independent cross-check that will become competitive in the coming years.

Here we present lattice QCD calculations of the LO-HVP contribution to the anomalous magnetic moments 
of all three leptons. The calculations include all contributions from $u, d, s$, and $c$ quarks, directly at the physical values of their masses, in their quark-connected and quarkdisconnected configurations. Contributions from third generation quarks can easily be estimated and are found to be much smaller than our statistical errors, even for the $\tau$ that is most sensitive to them (see, e.g., Ref. [40] for a calculation of the $b$ contribution to $a_{\mu}^{\mathrm{LO}-\mathrm{HVP}}$ ). Some previous lattice calculations of $a_{\ell}^{\mathrm{LO}-\mathrm{HVP}}, \ell=e, \mu[25,31]$, and of $a_{\ell}^{\mathrm{LO}-\mathrm{HVP}}, \ell=e$, $\mu, \tau$ [41], included all of these contributions, but involved difficult extrapolations to the physical value of the average $u$ - $d$ quark mass and only estimated the disconnected parts. In the present Letter, we work directly at the physical point and compute disconnected contributions directly. Moreover, we implement a description of the lattice results $[42,43]$ that solves the small virtuality issue [44] with finite-volume (FV) artifacts that are exponentially suppressed in lattice size.

A unified treatment of the HVP contribution to the three lepton anomalous magnetic moments provides important cross-checks that validate the methods used. As the typical virtualities probed by these moments are around $m_{\ell}^{2} / 4$, the vast difference in the mass of the leptons means that a large range of relevant scales are checked. In particular, agreement between our results and phenomenology in the electron case validates our understanding of small virtualities, and of larger virtualities in the $\tau$ case. In addition, the inclusion of all flavors up to the charm allows a controlled matching onto perturbation theory. Thus, all energy scales from zero to infinity are controlled in our calculation.

Methodology.-We consider the zero three-momentum two-point function of the quark electromagnetic current in Euclidean time $t$ :

$$
C_{\mu \nu}(t)=\frac{1}{e^{2}} \int d^{3} x\left\langle j_{\mu}(x) j_{\nu}(0)\right\rangle,
$$

with $e$ the positron charge, $x=(t, \vec{x})$ and $j_{\mu} / e=$ $\frac{2}{3} \bar{u} \gamma_{\mu} u-\frac{1}{3} \bar{d} \gamma_{\mu} d-\frac{1}{3} \bar{s} \gamma_{\mu} s+\frac{2}{3} \bar{c} \gamma_{\mu} c$. We work in the isospin limit, $m_{u}=m_{d}$. Because $C_{\mu \nu}$ 's flavor components are calculated separately and have different statistical and systematic uncertainties, it is useful to treat them separately. Physically, an isospin separation is useful. Thus,

$$
\begin{aligned}
C_{\mu \nu}(t) & =C_{\mu \nu}^{u d}(t)+C_{\mu \nu}^{s}(t)+C_{\mu \nu}^{c}(t)+C_{\mu \nu}^{\mathrm{disc}}(t) \\
& =C_{\mu \nu}^{I=1}(t)+C_{\mu \nu}^{I=0}(t),
\end{aligned}
$$

where in the top equality the first three terms correspond to the quark-connected contractions of the light ( $u$ and $d$ combined), strange and charm quarks, and the fourth to the quark-disconnected contractions of all four flavors. In the second equality, the separation is made between isospin $I=1$ and $I=0$ contributions, given by $C_{\mu \nu}^{I=1}=\frac{9}{10} C_{\mu \nu}^{u d}$ and $C_{\mu \nu}^{I=0}=\frac{1}{10} C_{\mu \nu}^{u d}+C_{\mu \nu}^{s}+C_{\mu \nu}^{c}+C_{\mu \nu}^{\text {disc }}$.

It is straightforward to obtain the corresponding LO-HVP contributions to the anomalous magnetic moment of lepton $\ell$ from these correlation functions $[21,45,46]$ :

$$
a_{\ell, f}^{\mathrm{LO}-\mathrm{HVP}}=\left(\frac{\alpha}{\pi}\right)^{2} \int_{0}^{\infty} \frac{d Q^{2}}{m_{\ell}^{2}} \omega\left(\frac{Q^{2}}{m_{\ell}^{2}}\right) \hat{\Pi}^{f}\left(Q^{2}\right),
$$

with $\quad \omega(r)=\pi^{2}[r+2-\sqrt{r(r+4)}]^{2} / \sqrt{r(r+4)}, \quad \alpha=$ $e^{2} /(4 \pi)$ and where the scalar polarization function renormalized in the Thomson limit is given by (see also Ref. [42])

$$
\begin{aligned}
\hat{\Pi}^{f}\left(Q^{2}\right) & \equiv \Pi^{f}\left(Q^{2}\right)-\Pi^{f}(0) \\
& =\frac{1}{3} \sum_{i=1}^{3} \int_{0}^{\infty} d t\left[t^{2}-\frac{4}{Q^{2}} \sin ^{2}\left(\frac{Q t}{2}\right)\right] \operatorname{Re} C_{i i}^{f}(t) .
\end{aligned}
$$

In Eqs. (3) and (4), the superscript $f$ can stand for $u d, s, c$, disc, $I=1, I=0$, and $\_$where the "." indicates that this equation also applies to the full LO-HVP contribution. Equation (4) implicitly includes the subtraction of the polarization tensor $\Pi_{\mu \nu}(Q=0)$, which was shown in Ref. [28] to be critical for reducing FV effects and, through the factor $t^{2}$, the subtraction of the polarization scalar $\Pi^{f}(0)$.

On a $T \times L^{3}$ lattice with spacing $a$, the integral over $t$ in Eq. (4) is replaced by a sum, in increments of $a$, that runs up to $T / 2$, once the correlator $C_{i i}^{f}(t)$ has been averaged with $C_{i i}^{f}(T-t)$. Moreover, the integral over $Q$ in Eq. (3) should, in principle, be replaced by a sum from 0 to $\pi / a$ in steps of $2 \pi / T$. Here we keep the integral, but cut it off at a value $Q=Q_{\max }$, chosen much smaller than $\pi / a$, so as to keep discretization errors under control, but above which perturbation theory can be applied. Then we decompose the anomalous magnetic moments of the leptons into three terms:

$$
\begin{aligned}
a_{\ell, f}^{\mathrm{LO}-\mathrm{HVP}}= & a_{\ell, f}^{\mathrm{LO}-\mathrm{HVP}}\left(Q \leq Q_{\max }\right)+\gamma_{\ell}\left(Q_{\max }\right) \hat{\Pi}^{f}\left(Q_{\max }^{2}\right) \\
& +\Delta^{\mathrm{pert}} a_{\ell, f}^{\mathrm{LO}-\mathrm{HVP}}\left(Q>Q_{\max }\right),
\end{aligned}
$$

where the low momentum contribution, $a_{\ell, f}^{\mathrm{LO}-\mathrm{HVP}}\left(Q \leq Q_{\max }\right)$, is obtained from the lattice as described above, and where the last term is the high-momentum, contribution renormalized at $Q_{\max }$ and computed in perturbation theory [47]. The second term in Eq. (5) is required to shift the renormalization point from $Q_{\max }$ to $Q=0$. It is obtained with lattice results for $C_{i i}^{f}(t)$, through Eq. (4) with $Q=Q_{\max } \cdot \gamma_{\ell}\left(Q_{\max }\right)$ is a known kinematical factor [47]. In obtaining Eq. (5), it is assumed that $\Delta^{\text {pert }} a_{\ell, f}^{\mathrm{LO}-\mathrm{HVP}}\left(Q>Q_{\max }\right)$ is equal to the value that it would have nonperturbatively. We check this by studying the dependence of our results on the choice of $Q_{\max }$.

The replacement of the FV sum over $Q$ by the corresponding integral is our choice of interpolation for the HVP function $\hat{\Pi}\left(Q^{2}\right)$. It constitutes an alternative to, e.g., the Padé approximation proposed in Ref. [44], the Marichev interpolation advocated in Ref. [48], or the finite-energy sum rule approach of Ref. [49]. The integrand in Eq. (3) has no singularities in the region of integration. Therefore, an application of Poisson's summation theorem guarantees that the corrections entailed in replacing the sum by an 
integral over $Q$ are exponentially small in $T$. These corrections will be accounted for in our estimate of $\mathrm{FV}$ uncertainties.

Lattice details.-We employ a tree-level improved Symanzik gauge action [50] and a fermion action for $N_{f}=$ $2+1+1$ flavors of stout-smeared [51], rooted, staggered quarks. We have generated 15 ensembles at six values of the bare coupling, $\beta$, corresponding to lattice spacings ranging from 0.064 to $0.134 \mathrm{fm}$. The average up and down quark mass and the strange quark mass are tuned to around the physical mass point defined using the Goldstone pion and kaon masses. The charm quark mass is fixed in units of the strange mass to $m_{c} / m_{s}=11.85$ [52]. The spatial dimensions of our lattices are in the range 6.1-6.6 fm and the temporal ones in the interval 8.6-11.3 fm. The lattice spacing is fixed with the pion leptonic decay constant, $f_{\pi}$. At each value of $\beta$, between 450 and 3500 configurations, separated by 10 unit length rational hybrid Monte Carlo (RHMC) [53] trajectories, are used. Details are given in Ref. [47] and more information about the simulations can be found in Ref. [54].

For the electromagnetic current correlator, we use the conserved lattice current at the source and sink so that no renormalization is necessary. We calculate the connected contributions to the correlators using point sources. We use the all-mode-averaging (AMA) technique of Ref. [55] and 768 random source positions on each configuration for the light quarks, 64 sources for the strange and 4 for the charm. To compute the quark-disconnected contributions, we apply AMA again, and exploit the approximate SU(3) flavor symmetry on around 6000 stochastic sources $[30,56]$. These are random, four-volume sources with which we compute the zero-momentum, time propagators, correcting for bias. For the disconnected contribution of the charm we apply a hopping parameter expansion.

Analysis.-Even with our high statistics, the signal deteriorates quickly with increasing distance in our light and disconnected correlators. Thus, in implementing Eqs. (3) and (4), we introduce cuts, $t_{c}$, in time beyond which we replace the correlator by the average of an upper and a lower bound [35,57]. $t_{c}$ is chosen such that the upper and lower bounds agree well within statistical errors and where these errors are not too large. The upshot is that our result for the light contribution to $a_{\ell}^{\mathrm{LO}-\mathrm{HVP}}$ is obtained by summing the integrand in Eq. (4) with $C_{i i}^{u d}(t)$ given by our lattice data up to $t_{c}$ and performing the rest of the sum from $t>t_{c}$ to $T / 2$ with $C_{i i}^{u d}(t)$ replaced by the bound average. The results of this procedure for $t_{c}$ in the range of $(3.000 \pm$ $0.134) \mathrm{fm}$ are averaged to account for possible statistical fluctuations in the correlator at a given $t_{c}$. The disconnected contribution to $a_{\ell}^{\mathrm{LO}-\mathrm{HVP}}$ is obtained in an identical fashion, but with $t_{c}$ in the range $(2.600 \pm 0.134) \mathrm{fm}$.

We limit the integral over $Q$ in Eq. (3) to $Q_{\max }$, and use perturbation theory to obtain the complement. We consider $Q_{\max }^{2}=1,2, \ldots, 5 \mathrm{GeV}^{2}$. In what follows, quantities with the subscript "lat" correspond to lattice results obtained in a given simulation. Their dependence on lattice spacing and quark masses will be left implicit. To extrapolate our results $a_{\ell, f, \text { lat }}^{\mathrm{LO}}\left(Q \leq Q_{\max }\right)$ to the continuum limit and to interpolate them to the physical mass point, we fit them to a function which depends on the Goldstone pion and kaon masses squared, on the $\eta_{c}$ mass and on the lattice spacing squared [59]. Since the simulations are performed close to the physical mass point, a constant or linear dependence in the mass parameters is always sufficient. Moreover, for all flavor contributions, good fit qualities can be achieved with a linear $a^{2}$ dependence for all three leptons and all values of $Q_{\max }$ considered here. Because taste violations play an important role in the continuum extrapolation of $a_{\ell, u d}^{\mathrm{LO}-\mathrm{HVP}}$, we have also tried correcting for these effects using oneloop staggered chiral perturbation theory before performing a continuum extrapolation [34]. While the continuum extrapolation is significantly milder, the continuum limit results obtained are consistent with the ones presented here. Our continuum extrapolations are discussed in detail and examples are shown in Ref. [47]. Here we emphasize that with simulations at six lattice spacings down to $0.064 \mathrm{fm}$, we have full control over the continuum extrapolations.

This analysis yields the continuum extrapolated flavor quantities, $a_{\ell, f}^{\mathrm{LO}-\mathrm{HVP}}\left(Q \leq Q_{\max }\right)$, for the five values of $Q_{\max }$ considered. For each value of $Q_{\max }$, we sum the appropriate flavor quantities, to get the corresponding $I=1, I=0$, and total, low- $Q$ contributions to the lepton anomalous magnetic moments. The results for these and the individual flavor contributions are given in Ref. [47], with statistical and systematic errors obtained as described below. To these contributions we add the corresponding complements given in Eq. (5). These complements require the computation of $\hat{\Pi}^{f}\left(Q_{\max }^{2}\right)$. This is done using Eq. (4) and requires a continuum limit and physical mass point interpolation very similar to that performed for $a_{\ell, f, \text { lat }}^{\mathrm{LO}-\mathrm{HVP}}\left(Q \leq Q_{\max }\right)$ [47].

The perturbative contributions, $\Delta^{\text {pert }} a_{\ell, f}^{\mathrm{LO}-\mathrm{HVP}}\left(Q>Q_{\max }\right)$, are computed from results for $\Pi^{f}\left(Q^{2}\right)$, with terms up to $O\left(\alpha_{s}^{4}\right)$, obtained using the code rhad [69], as explained in Ref. [47]. These corrections are below our statistical errors for the $e$ and $\mu$, which have very little sensitivity to large $Q$, but are significant for the $\tau$. In Ref. [47] we study the $Q_{\max }$ dependence of our results for $a_{\ell, f}^{\mathrm{LO}-\mathrm{HVP}}$. The fact that they are independent of $Q_{\max } \geq \sqrt{2} \mathrm{GeV}$ within errors, in particular for $\ell=\tau$, indicates that our continuum-limit, lattice results are consistent with five-loop perturbation theory for momenta, $Q$, above that value.

Systematic errors and results. - The procedure described above yields $a_{\ell, f}^{\mathrm{LO}-\mathrm{HVP}}$ for all $f$ and for all three leptons. In our physical fits, the errors associated with the small interpolations in mass are negligible. Those associated with the continuum extrapolations are not. To estimate them, we impose four cuts on the lattice spacing: no cut, and $a \leq 0.118,0.111,0.095 \mathrm{fm}$. This number is reduced to 
TABLE I. LO-HVP contribution to the anomalous magnetic moments of the $e, \mu$, and $\tau$ leptons. The first two lines give our results for the $I=1$ and $I=0$ contributions. The $I=1$ results include the FV corrections, which are negligible in the $I=0$ case. The last line displays our results for the total LO-HVP contribution. In addition to the terms included in the $I=1$ and $I=0$ components, this total also accounts for QED and $m_{d} \neq m_{u}$ corrections. The first error on all results is statistical, the second is associated with the continuum extrapolation, the third with our bounding procedure, the fourth with the matching to perturbation theory, the fifth with the lattice spacing uncertainty and, where applicable, the sixth with the FV correction and the seventh with the IB correction.

\begin{tabular}{lccc}
\hline \hline & $\ell=e$ (units of $\left.10^{-14}\right)$ & $\ell=\mu\left(\right.$ units of $\left.10^{-10}\right)$ & $\ell=\tau\left(\right.$ units of $\left.10^{-8}\right)$ \\
\hline$a_{\ell, I=1}^{\mathrm{LO}-\mathrm{HVP}}$ & $156.9(2.4)(2.1)(0.0)(0.0)(1.2)(4.6)$ & $582.9(6.7)(7.2)(0.1)(0.0)(4.5)(13.5)$ & $253.2(0.7)(1.4)(0.0)(0.1)(1.2)(1.8)$ \\
$a_{\ell, I=0}^{\mathrm{LO}-\mathrm{HVP}}$ & $30.7(1.2)(1.0)(0.1)(0.0)(0.2)$ & $120.5(3.4)(3.5)(0.2)(0.0)(1.0)$ & $84.4(0.4)(0.7)(0.0)(1.1)(3.4)$ \\
$a_{\ell}^{\mathrm{LO}-\mathrm{HVP}}$ & $189.3(2.6)(2.3)(0.1)(0.0)(1.5)(4.6)(1.6)$ & $711.1(7.5)(8.0)(0.2)(0.0)(5.5)(13.5)(5.1)$ & $341.0(0.8)(1.6)(0.0)(1.1)(1.5)(1.8)(1.1)$ \\
\hline \hline
\end{tabular}

three in the disconnected case for which we have no results at $a=0.064 \mathrm{fm}$. The systematic error associated with the matching to perturbation theory is determined from the results with $Q_{\max }^{2}=2, \ldots, 5 \mathrm{GeV}^{2}$, covering the range safely accessible to our lattice calculations and to perturbation theory. The one associated with the time cut is determined by considering $t_{c}$ ranges shifted by $-0.134 \mathrm{fm}$ compared to those given above. Over the total range of $t_{c}$ considered, the two-pion bounds change by a factor close to 3 . The final central value is the unweighted average of all results. Each systematic error component is chosen to cover all of the central values resulting from the variation, over the ranges described above, of the variable associated with that component. Furthermore, we add a $0.8 \%$ systematic error to our results for $a_{\ell, f}^{\mathrm{LO}-\mathrm{HVP}}\left(Q \leq Q_{\max }\right)$ due to the uncertainty in our determination of the lattice spacing [47]. The statistical error is the jackknife error of the central value over jackknife samples with bins of length 10 configurations. The results for the individual flavor contributions to the magnetic moments of all three leptons are given in Ref. [47].

In the absence of a systematic study with simulations in a variety of volumes, only model estimates of FV effects can be made. As argued in Refs. [70,71], for large volumes those effects will be governed by pion contributions that can be computed in chiral perturbation theory $(\chi \mathrm{PT})[70]$. Since the $I=0$ channel is dominated by three-pion exchange, the FV effects are expected to be smaller than those of the $I=1$ contribution, which are already small. Thus we consider only the latter. Our computation of these effects is summarized in Ref. [47] and the appropriate corrections are added to our $I=1$ and total results. They are 4.6(4.6) $\times 10^{-14}$ for the $e$ and $13.5(13.5) \times 10^{-10}$ for the $\mu$ with negligible $Q_{\max }$ dependence in the range of interest. For the $\tau$ they range from $9.4(9.4) \times 10^{-9}$ to $1.6(1.6) \times 10^{-8}$ for $Q_{\max }=1 \div \sqrt{5} \mathrm{GeV}$. We associate with these corrections a $100 \%$ uncertainty included in our error budget.

Compared to phenomenological determinations of $a_{\mu}^{\mathrm{LO}-\mathrm{HVP}}[8,19,20]$, our $m_{d}=m_{u}$ calculation without QED is missing isospin-breaking (IB) effects. These are detailed in Ref. [47]. Here we note that the corrections to be added are $(1.7 \pm 1.6) \times 10^{-14}$ for the $e,(7.8 \pm 5.1) \times$ $10^{-10}$ for the $\mu$, and $(3.4 \pm 1.1) \times 10^{-8}$ for the $\tau$.
We quote our final results for $a_{\ell}^{\mathrm{LO}-\mathrm{HVP}}$ for all three leptons in Table I. Combining all errors in quadrature, we obtain $a_{e}^{\mathrm{LO}-\mathrm{HVP}}$ with an uncertainty of $3.3 \%, a_{\mu}^{\mathrm{LO}-\mathrm{HVP}}$ of $2.7 \%$ and $a_{\tau}^{\mathrm{LO}-\mathrm{HVP}}$ of $1.0 \%$. Not surprisingly, the relative error increases with the sensitivity of the anomalous moment to long-distance physics.

Discussion.-It is interesting to compare these results with those in the literature. There are only two lattice QCD determinations of the LO-HVP contribution to the muon anomalous moment which include the contributions of quarks up to the charm [25,34]. Compared to those, our calculation is the only one in which the continuum extrapolation is performed directly at the physical mass point and which includes a reliable determination of the quark-disconnected contribution. There exist also many precise phenomenological determinations of $a_{\mu}^{\mathrm{LO}-\mathrm{HVP}}$, as discussed in the Introduction. Here we consider three recent ones $[8,19,20]$.

We plot all of these results in Fig. 1, together with ours. Also shown on this plot is the value that $a_{\mu}^{\mathrm{LO}-\mathrm{HVP}}$ would have to have to explain the experimental measurement

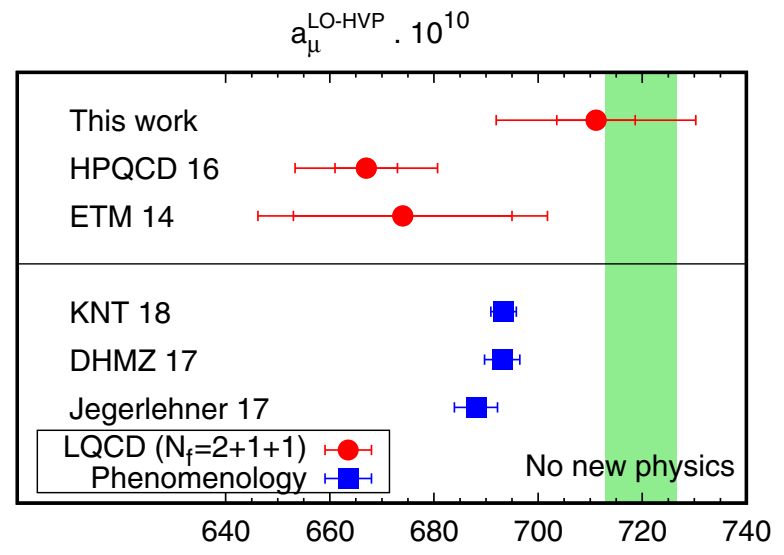

FIG. 1. Comparison of our result for $a_{\mu}^{\mathrm{LO}-\mathrm{HVP}}$ with the only other two $N_{f}=2+1+1$ lattice QCD calculations [25,34] and with recent ones obtained from phenomenology $[8,19,20]$. For the lattice results, the first error is statistical and the second is the total error, including systematics. The shaded region is the value that $a_{\mu}^{\mathrm{LO}-\mathrm{HVP}}$ would have to have to explain the experimental measurement of $a_{\mu}$, assuming no new physics. 
of $a_{\mu}$ [7], assuming that all other SM contributions are unchanged, i.e., assuming no new physics (NP). Using the SM contributions summarized in Ref. [8], we find $a_{\mu, \text { noNP }}^{\mathrm{LO}}=(720.0 \pm 6.8) \times 10^{-10}$. The errors on the lattice results, which are in the range of $2.0 \%$ to $4.1 \%$ are substantially larger than those of the phenomenological approach. Our result for $a_{\mu}^{\mathrm{LO}-\mathrm{HVP}}$ is larger than those of the other lattice calculations and in slight tension with the one from HPQCD [34] which is $1.9 \sigma$ away. A more detailed flavor-by-flavor comparison is given in Ref. [47]. However, our result is consistent with those from phenomenology within about 1 standard deviation, as well as with $a_{\mu, \text { noNP }}^{\mathrm{LO}}$. Thus, one will have to wait for the next generation of lattice QCD calculations to confirm or infirm the larger than $3 \sigma$ deviation between the measurement of $a_{\mu}$ and the prediction of the SM based on phenomenology.

Regarding $a_{e}^{\mathrm{LO}-\mathrm{HVP}}$, there are two other lattice calculations $[34,41]$ and only one concerning $a_{\tau}^{\mathrm{LO}-\mathrm{HVP}}$ [41]. The results in Ref. [41] are $a_{e}^{\mathrm{LO}-\mathrm{HVP}}=1.782(64)(86) \times 10^{-12}$ and $a_{\tau}^{\mathrm{LO}-\mathrm{HVP}}=3.41(8)(6) \times 10^{-6}$ and in Ref. [34], $a_{e}^{\mathrm{LO}-\mathrm{HVP}}=$ $1.779(39) \times 10^{-12}$. From the point of view of phenomenology, a dispersive analysis similar to the one implemented for the muon gives $a_{e}^{\mathrm{LO}-\mathrm{HVP}}=1.846(12) \times 10^{-12}[14]$ and $a_{\tau}^{\mathrm{LO}-\mathrm{HVP}}=3.38(4) \times 10^{-6}$ [13]. Comparing these results to ours in Table I, we find the following. The result of Ref. [34] for $a_{e}^{\mathrm{LO}-H V P}$ displays a tension with ours which is slightly smaller than the one for $a_{\mu}^{\mathrm{LO}-\mathrm{HVP}}$. On the other hand, our results are fully compatible with the phenomenological ones, indicating that we control the physics of the HVP over full range of $Q^{2}$. In addition, our result for $a_{e}^{\mathrm{LO}-\mathrm{HVP}}$ has an error which is about half that of the lattice result of Ref. [41] and for $a_{\tau}^{\mathrm{LO}-\mathrm{HVP}}$, it is approximately 3 times more precise. In fact, our result for the latter is more precise than the phenomenological one.

We thank S. Dürr for contributions in the early stages of this project and Z. Zhang for noticing a typo in our final result for $a_{e}^{\mathrm{LO}-\mathrm{HVP}}$. In addition, L. L. thanks M. Benayoun, C. Davies, F. Jegerlehner, M. Knecht, C. Lehner, E. de Rafael, and R. Van de Water for informative discussions. Computations were performed on JUQUEEN and JUROPA at Forschungszentrum Jülich, on Turing at the Institute for Development and Resources in Intensive Scientific Computing (IDRIS) in Orsay, on SuperMUC at Leibniz Supercomputing Centre in München, on Hermit at the High Performance Computing Center in Stuttgart. This project was supported, in part, by the OCEVU Laboratoire d'excellence (ANR-11-LABX-0060) and the A*MIDEX Project (ANR-11-IDEX-0001-02), which are funded by the "Investissements d'avenir" French government program and managed by the "Agence nationale de la recherche" (ANR), by the DFG Grant No. SFB/TR55, by the Gauss Centre for Supercomputing e.V and by the GENCI-IDRIS supercomputing Grant No. 52275. R. M. was supported in part by a joint Ph.D. fellowship from the Centre national de la recherche scientifique (CNRS) and the Commissariat à l'énergie atomique et aux énergies alternatives (CEA).

Note added.-Recently, a work reporting on an $N_{f}=2+1$ lattice QCD calculation of $a_{\mu}^{\mathrm{LO}-\mathrm{HVP}}$ appeared [72]. That calculation includes a lattice computation of many isospin breaking effects. Its result for $a_{\mu}^{\mathrm{LO}-\mathrm{HVP}}$ is in excellent agreement with ours.

*Corresponding author. lellouch@cpt.univ-mrs.fr

[1] W. Gerlach and O. Stern, Z. Phys. 8, 110 (1922).

[2] S. A. Goudschmidt and G. H. Uhlenbeck, Nature (London) 117, 264 (1926).

[3] F. Jegerlehner and A. Nyffeler, Phys. Rep. 477, 1 (2009).

[4] D. Hanneke, S. Fogwell, and G. Gabrielse, Phys. Rev. Lett. 100, 120801 (2008).

[5] T. Aoyama, M. Hayakawa, T. Kinoshita, and M. Nio, Phys. Rev. Lett. 109, 111808 (2012).

[6] T. Aoyama, M. Hayakawa, T. Kinoshita, and M. Nio, Phys. Rev. D 91, 033006 (2015).

[7] G. W. Bennett et al. (Muon g-2 Collaboration), Phys. Rev. D 73, 072003 (2006).

[8] M. Davier, A. Hoecker, B. Malaescu, and Z. Zhang, Eur. Phys. J. C 77, 827 (2017).

[9] J. L. Holzbauer, in Proceedings, 12th International Conference on Beauty, Charm, and Hyperons in Hadronic Interactions (BEACH 2016): Fairfax, Virginia, USA, 2016, [J. Phys. Conf. Ser. 770, 012038 (2016)].

[10] M. Otani (E34 Collaboration), in Proceedings, 2nd International Symposium on Science at J-PARC: Unlocking the Mysteries of Life, Matter and the Universe (J-PARC 2014): Tsukuba, Japan, 2014, [J. Phys. Soc. Jpn. Conf. Proc. 8, 025008 (2015)].

[11] M. Fael, L. Mercolli, and M. Passera, in Proceedings, 12th International Workshop on Tau Lepton Physics (TAU 2012): Nagoya, Japan, 2012, [Nucl. Phys. B, Proc. Suppl. 253-255, 103 (2014)].

[12] B. Oberhof (BABAR Collaboration), in Proceedings, 13th International Workshop on Tau Lepton Physics (TAU 2014): Aachen, Germany, 2014, [Nucl. Part. Phys. Proc. 260, 12 (2015)].

[13] S. Eidelman and M. Passera, Mod. Phys. Lett. A 22, 159 (2007).

[14] F. Jegerlehner, in Proceedings, Workshop on Flavour changing and conserving processes 2015 (FCCP2015), [Eur. Phys. J. Web Conf. 118, 01016 (2016)].

[15] S. Eidelman and F. Jegerlehner, Z. Phys. C 67, 585 (1995).

[16] M. Davier, A. Hoecker, B. Malaescu, and Z. Zhang, Eur. Phys. J. C 71, 1515 (2011); 72, 1874(E) (2012).

[17] K. Hagiwara, R. Liao, A. D. Martin, D. Nomura, and T. Teubner, J. Phys. G 38, 085003 (2011).

[18] J. P. Miller, E. de Rafael, B. L. Roberts, and D. Stöckinger, Annu. Rev. Nucl. Part. Sci. 62, 237 (2012).

[19] F. Jegerlehner, in KLOE-2 workshop on $e^{+} e^{-}$collider physics at $1 \mathrm{GeV}$, INFN-Laboratori Nazionali di Frascati, Italy, 2016, [Eur. Phys. J. Web Conf. 166, 00022 (2018)]. 
[20] A. Keshavarzi, D. Nomura, and T. Teubner, arXiv: 1802.02995 [Phys. Rev. D (to be published)].

[21] T. Blum, Phys. Rev. Lett. 91, 052001 (2003).

[22] C. Aubin and T. Blum, Phys. Rev. D 75, 114502 (2007).

[23] X. Feng, K. Jansen, M. Petschlies, and D. B. Renner, Phys. Rev. Lett. 107, 081802 (2011).

[24] M. D. Morte, B. Jager, A. Juttner, and H. Wittig, J. High Energy Phys. 03 (2012) 055.

[25] F. Burger, X. Feng, G. Hotzel, K. Jansen, M. Petschlies, and D. B. Renner (ETM Collaboration), J. High Energy Phys. 02 (2014) 099.

[26] T. Blum, M. Hayakawa, and T. Izubuchi, Proc. Sci., LATTICE2012 (2012) 022 [arXiv:1301.2607].

[27] E. B. Gregory, Z. Fodor, C. Hoelbling, S. Krieg, L. Lellouch, R. Malak, C. McNeile, and K. Szabo, Proc. Sci., LATTICE2013 (2014) 302 [arXiv:1311.4446].

[28] R. Malak, Z. Fodor, C. Hoelbling, L. Lellouch, A. Sastre, and K. Szabo, Proc. Sci., LATTICE2014 (2015) 161 [arXiv:1502.02172].

[29] B. Chakraborty , C. T. H. Davies, G. C. Donald, R. J. Dowdall, J. Koponen, G. P. Lepage, and T. Teubner (HPQCD Collaboration), Phys. Rev. D 89, 114501 (2014).

[30] T. Blum, P. A. Boyle, T. Izubuchi, L. Jin, A. Jüttner, C. Lehner, K. Maltman, M. Marinkovic, A. Portelli, and M. Spraggs, Phys. Rev. Lett. 116, 232002 (2016).

[31] B. Chakraborty, C. T. H. Davies, J. Koponen, G. P. Lepage, M. J. Peardon, and S. M. Ryan, Phys. Rev. D 93, 074509 (2016).

[32] G. S. Bali and G. Endrődi, Phys. Rev. D 92, 054506 (2015).

[33] T. Blum et al. (RBC and UKQCD Collaborations), J. High Energy Phys. 04 (2016) 063; 05 (2017) 34.

[34] B. Chakraborty, C. T. H. Davies, P. G. de Oliveira, J. Koponen, G. P. Lepage, and R. S. Van de Water, Phys. Rev. D 96, 034516 (2017).

[35] S. Borsanyi, Z. Fodor, T. Kawanai, S. Krieg, L. Lellouch, R. Malak, K. Miura, K. K. Szabo, C. Torrero, and B. C. Toth (Budapest-Marseille-Wuppertal Collaboration), Phys. Rev. D 96, 074507 (2017).

[36] M. Della Morte, A. Francis, V. Gülpers, G. Herdoíza, G. von Hippel, H. Horch, B. Jäger, H. B. Meyer, A. Nyffeler, and H. Wittig, J. High Energy Phys. 10 (2017) 020.

[37] D. Giusti, V. Lubicz, G. Martinelli, F. Sanfilippo, and S. Simula, J. High Energy Phys. 10 (2017) 157.

[38] P. Boyle, V. Gülpers, J. Harrison, A. Jüttner, C. Lehner, A. Portelli, and C. T. Sachrajda, J. High Energy Phys. 09 (2017) 153.

[39] B. Chakraborty, C. T. H. Davies, C. DeTar, A. X. El-Khadra, E. Gámiz, S. Gottlieb, D. Hatton, J. Koponen, A. S. Kronfeld, J. Laiho, G. P. Lepage, Y. Liu, P. B. Mackenzie, C. McNeile, E. T. Neil, J. N. Simone, R. Sugar, D. Toussaint, R. S. V. de Water, and A. Vaquero, Phys. Rev. Lett. 120, 152001 (2018).

[40] B. Colquhoun, R. J. Dowdall, C. T. H. Davies, K. Hornbostel, and G. P. Lepage, Phys. Rev. D 91, 074514 (2015).

[41] F. Burger, K. Jansen, M. Petschlies, and G. Pientka, Eur. Phys. J. C 76, 464 (2016).
[42] D. Bernecker and H. B. Meyer, Eur. Phys. J. A 47, 148 (2011).

[43] M. Spraggs, P. Boyle, L. Del Debbio, A. Jüttner, C. Lehner, K. Maltman, M. Marinkovic, and A. Portelli, Proc. Sci., LATTICE2015 (2016) 106 [arXiv:1601.00537].

[44] C. Aubin, T. Blum, M. Golterman, and S. Peris, Phys. Rev. D 86, 054509 (2012).

[45] B. e. Lautrup, A. Peterman, and E. de Rafael, Phys. Rep. 3, 193 (1972).

[46] E. de Rafael, Phys. Lett. B 322, 239 (1994).

[47] See Supplemental Material at http://link.aps.org/ supplemental/10.1103/PhysRevLett.121.022002 for details and further explanations of the issues discussed in the sentences preceding the present reference.

[48] E. de Rafael, Phys. Rev. D 96, 014510 (2017).

[49] C. A. Dominguez, K. Schilcher, and H. Spiesberger, Nuovo Cim. C 40, 179 (2017).

[50] M. Luscher and P. Weisz, Commun. Math. Phys. 97, 59 (1985); 98, 433(E) (1985).

[51] C. Morningstar and M. J. Peardon, Phys. Rev. D 69, 054501 (2004).

[52] C. T. H. Davies, C. McNeile, K. Y. Wong, E. Follana, R. Horgan, K. Hornbostel, G. P. Lepage, J. Shigemitsu, and H. Trottier, Phys. Rev. Lett. 104, 132003 (2010).

[53] M. A. Clark and A. D. Kennedy, Phys. Rev. Lett. 98, 051601 (2007).

[54] R. Bellwied, S. Borsanyi, Z. Fodor, S. D. Katz, A. Pasztor, C. Ratti, and K. K. Szabo, Phys. Rev. D 92, 114505 (2015).

[55] T. Blum, T. Izubuchi, and E. Shintani, Phys. Rev. D 88, 094503 (2013).

[56] V. Gülpers, A. Francis, B. Jäger, H. Meyer, G. von Hippel, and H. Wittig, Proc. Sci., LATTICE2014 (2014) 128 [arXiv:1411.7592].

[57] See Supplemental Material at http://link.aps.org/ supplemental/10.1103/PhysRevLett.121.022002 for a discussion of our bounding procedure which includes a reference to Ref. [58].

[58] M. Lüscher, Nucl. Phys. B364, 237 (1991).

[59] See Supplemental Material at http://link.aps.org/ supplemental/10.1103/PhysRevLett.121.022002 for a more detailed explanation of how we define the physical point. This discussion includes references to Refs. [60-68].

[60] S. Aoki et al., Eur. Phys. J. C 77, 112 (2017).

[61] B. Chakraborty, C. T. H. Davies, B. Galloway, P. Knecht, J. Koponen, G. C. Donald, R. J. Dowdall, G. P. Lepage, and C. McNeile, Phys. Rev. D 91, 054508 (2015).

[62] J. Gasser and G. R. S. Zarnauskas, Phys. Lett. B 693, 122 (2010).

[63] M. Lüscher, J. High Energy Phys. 08 (2010) 071.

[64] S. Borsanyi et al. (Budapest-Marseille-Wuppertal Collaboration), J. High Energy Phys. 09 (2012) 010.

[65] J.S. Bell and E. de Rafael, Nucl. Phys. 11B, 611 (1969).

[66] J. L. Rosner, S. Stone, and R. S. Van de Water, arXiv: 1509.02220 [Particle Data Book (to be published)].

[67] C. Patrignani et al. (Particle Data Group), Chin. Phys. C 40, 100001 (2016). 
[68] R. J. Dowdall, C. T. H. Davies, G. P. Lepage, and C. McNeile, Phys. Rev. D 88, 074504 (2013).

[69] R. V. Harlander and M. Steinhauser, Comput. Phys. Commun. 153, 244 (2003).

[70] C. Aubin, T. Blum, P. Chau, M. Golterman, S. Peris, and C. Tu, Phys. Rev. D 93, 054508 (2016).
[71] A. Francis, B. Jager, H. B. Meyer, and H. Wittig, Phys. Rev. D 88, 054502 (2013).

[72] T. Blum, P. A. Boyle, V. Gülpers, T. Izubuchi, L. Jin, C. Jung, A. Jüttner, C. Lehner, A. Portelli, and J. T. Tsang, following Letter, Phys. Rev. Lett. 121, 022003 (2018). 